\title{
MINIREVIEW
}

\section{Acoel and platyhelminth models for stem-cell research}

\author{
Alexandra E Bely* and James M Sikes* \\ See research article http://www.biomedcentral.com/1471-213X/9/69
}

\begin{abstract}
Acoel and platyhelminth worms are particularly attractive invertebrate models for stem-cell research because their bodies are continually renewed from large pools of somatic stem cells. Several recent studies, including one in BMC Developmental Biology, are beginning to reveal the cellular dynamics and molecular basis of stem-cell function in these animals.
\end{abstract}

Adult somatic stem cells can play critical roles in postembryonic developmental processes such as tissue renewal, growth, repair, and regeneration [1]. Understanding how such cells are maintained and produce differentiated progeny is thus of general interest in developmental biology, in addition to being of clear biomedical relevance. Invertebrate models have great potential for elucidating the cellular and molecular basis of stem-cell function. However, in the main invertebrate models used for dissecting the details of animal development, including Drosophila and Caenorhabditis, adult somatic tissues are primarily post-mitotic and are largely or entirely devoid of adult stem cells, which limits the use of these established models for stem-cell research. Representatives of two groups of soft-bodied worms, the Acoela and the Platyhelminthes, possess large pools of adult somatic stem cells, making them useful invertebrate models for stem-cell biology. These organisms are now beginning to provide new insights into the cellular and molecular basis of adult stem-cell function.

*Correspondence: Alexandra E Bely. Email: abely@umd.edu; James M Sikes. Email: jsikes@illinois.edu

'Biology Department, University of Maryland, College Park, MD 20742, USA 2Department of Cell and Developmental Biology, University of Illinois, Urbana, IL 61801, USA

Full list of author information is available at the end of the article

\section{A remarkable stem-cell system in platyhelminth and acoel worms}

Thomas Hunt Morgan's classic experiments on the amazing regenerative abilities of planarians (phylum Platyhelminthes) helped fuel the early study of adult stem cells. In particular, these studies ultimately led to the discovery that planarians possess a very unusual stemcell system [2]: the body of a planarian is continually rebuilt from a large pool of somatic stem cells, called neoblasts, that are distributed throughout the animal. Neoblasts are the only mitotically active cells in the body and constantly proliferate to renew all cell types. Neoblasts are thus required for whole-body homeostasis and are likewise responsible for forming new tissues by growth and regeneration in these animals. Other platyhelminths also possess a similar stem-cell system, including one of the most basal lineages within the phylum, the macrostomids [3]. Thus, it is likely that possession of neoblasts is ancestral for the Platyhelminthes.

Although unusual among animals in general, a neoblast stem cell system is also known from a group of small, soft-bodied marine worms known as acoels. In a recent study published in BMC Developmental Biology, De Mulder et al. [4] present the first detailed characterization of this stem cell system in acoels. Working with the acoel Isodiametra pulchra, De Mulder et al. investigated neoblast distribution and proliferation using morphological analyses and S-phase cell labelling and also characterized in these animals the expression and function of a conserved stem-cell regulator.

Acoels have historically been placed within the Platyhelminthes, but recent molecular phylogenetic analyses now suggest that they probably represent a distinct phylum, the Acoela, that falls well outside of the Platyhelminthes (see [5] and references therein). Specifically, acoels are now thought to represent the most basal lineage within the Bilateria, being the sister group to all other bilaterian animals (Figure 1a). That a similar neoblast system is found in both Platyhelminthes and Acoela has important implications for the evolution of 


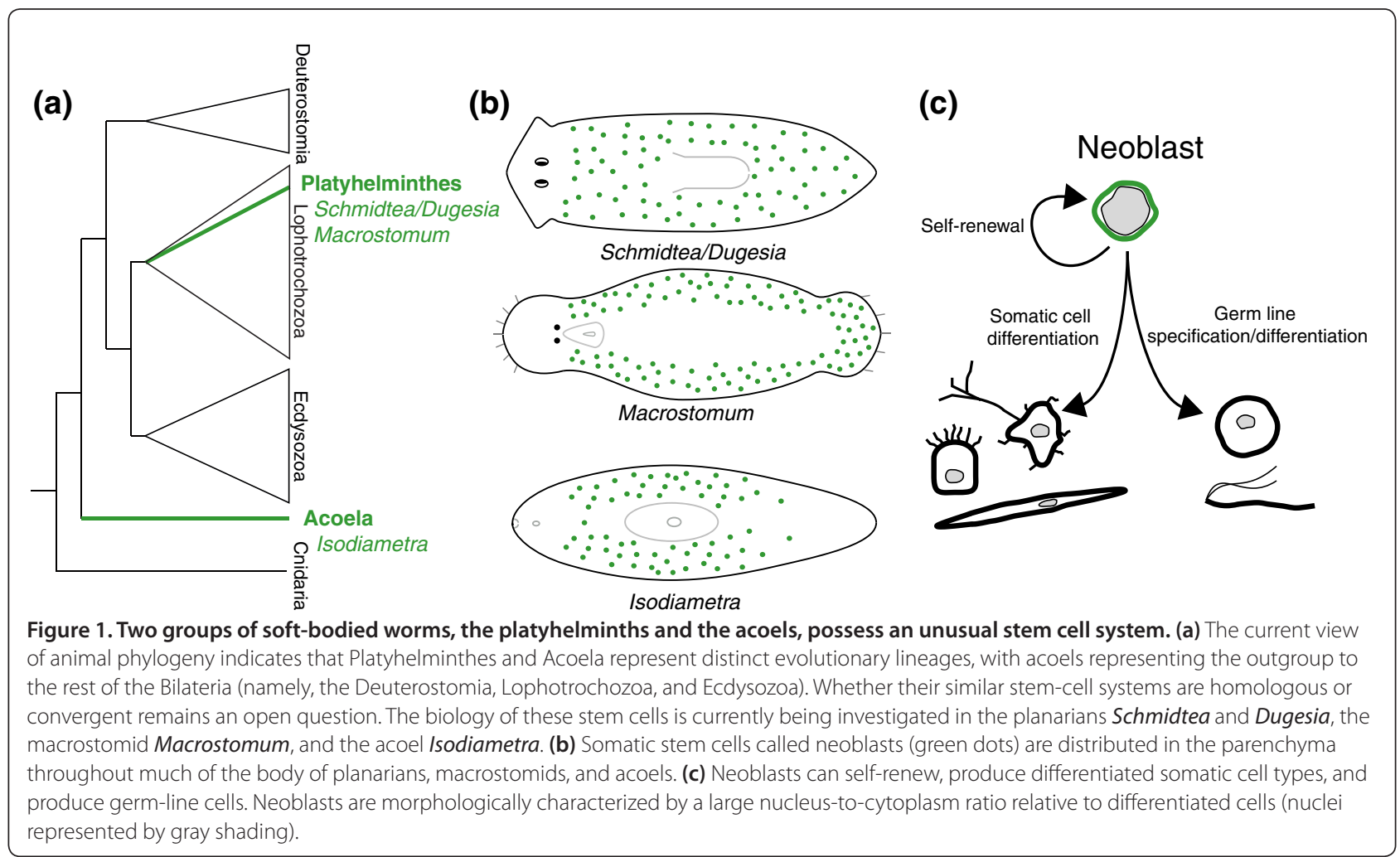

this unusual mode of homeostasis. If acoels are indeed the outgroup to all other bilaterians, the neoblast stemcell system may have evolved convergently in acoels and platyhelminths or, alternatively, may even be ancestral for all bilaterian animals.

Because of their unusual neoblast system, platyhelminths and acoels are particularly attractive invertebrate models for stem-cell research. They present a number of advantages, including the fact that a large pool of stem cells is available throughout the lifetime of each individual; the stem-cell pool is collectively totipotent, not just pluripotent (neoblasts can even give rise to the germ line); these stem cells exhibit high rates of turnover, undergoing continual self-renewal and production of differentiated progeny; and neoblasts are the only proliferative cells in the body while the rest of the body is post-mitotic, making it possible to selectively disrupt large pools of stem cells in vivo through wholebody irradiation or other techniques.

\section{Cellular and molecular dynamics of neoblasts}

Several platyhelminth and acoel species are being developed into powerful models for investigating stem cell biology. Although Dugesia was Morgan's original planarian subject, species in the closely related genus Schmidtea have rapidly become the best characterized of the planarians. As the research community working on this genus has grown, robust techniques have become available for marking and manipulating stem cells in this group and important genomic tools have been developed, including a fully sequenced genome for $S$. mediterranea (reviewed in [1]). Smaller research communities have also begun working on the stem cell biology of the macrostomid Macrostomum lignano and, most recently, the acoel Isodiametra pulchra. Collectively, these studies are beginning to reveal the generalities as well as the unique properties of this similar neoblast stem-cell system employed in different animal groups.

The in vivo cellular dynamics of neoblasts are remarkably similar in platyhelminths and acoels. In planarians, macrostomids, and acoels, neoblasts reside exclusively within the parenchyma, although the exact spatial distribution of neoblasts within the body varies slightly among these different groups (Figure 1b) $[4,6,7]$. Neoblast progeny then disperse out from the parenchyma, giving rise to new differentiated cells of the body. A recent microarray study in planarians indicates that the self-renewing neoblast population has a specific transcriptional profile and that the recent progeny of neoblasts express a sequence of distinct transcriptional profiles as they migrate and begin to differentiate [7]. Interestingly, in that study, several chromatin-modifying factors were found to be expressed in neoblasts, consistent with the idea that chromatin modification may 
play an important role in permitting cells to maintain an undifferentiated state.

Because neoblasts are the only proliferative cells within the body, irradiation that kills proliferating cells selectively destroys neoblasts. Studies carried out decades ago showed that in planarians, destroying the neoblast population of adults by whole-body irradiation results in gradual malformation of the body (as tissues fail to be renewed) and eventually death [2]. Destruction of the neoblast pool in planarians also abrogates the typically extensive regenerative ability of these animals. The study of De Mulder et al. demonstrates that in acoels irradiation dramatically reduces cell proliferation, abolishes the expression of a stem cell marker, and ultimately leads to death [4], indicating that neoblasts are required for homeostasis in acoels, as they are platyhelminths. The selective destruction of neoblasts by irradiation is a powerful tool in these animals. For example, a key aspect of the microarray study described above was the comparison of irradiated and unirradiated planarian tissue to reveal neoblast-specific genes [7].

\section{PIWI gene expression and function in neoblasts}

Robust methods for in situ hybridization and gene knockdown via RNA interference (RNAi) have now been developed for both platyhelminths and acoels, and these are providing insight into the molecular basis of the various functions of neoblasts $[1,4,8]$ (Figure 1c). One group of genes, the PIWI genes, has received particular attention as conserved regulators of stem-cell function. PIWI genes are a subfamily of the PIWI/Argonaute gene family and, in most animals investigated, are expressed specifically in the germ line, where they are thought to function in silencing transposons and translational gene regulation. In Schmidtea and Macrostomum, transcripts of piwi homologs are present not only in the germ line but also in the neoblasts, and gene knockdown by RNAi results in the eventual loss of both the germ-line and the neoblast pool [6,9]. De Mulder et al. [4] now find that a piwi homolog is also expressed in both germ line and neoblasts in Isodiametra. RNAi knockdown of its expression causes loss of the germ line but, unexpectedly, does not affect the neoblast pool or its proliferation. Whether there are other piwi homologs expressed redundantly in the neoblasts of this species remains to be determined.

Although the pattern of piwi homolog gene transcription in Schmidtea, Macrostomum and Isodiametra, coupled with the RNAi phenotype (neoblast depletion) in the first two, might suggest that piwi homologs are involved specifically in the self-renewal of neoblasts, this may not actually be the case. PIWI protein distribution has been characterized in both Schmidtea and Isodiametra, and in these animals PIWI protein is detected not only in neoblasts but also in recent neoblast progeny that are committed to differentiate $[4,10]$. Furthermore, the RNAi phenotype of at least one planarian piwi homolog indicates that neoblasts can persist and proliferate for many days following RNAi treatment, and that during this time their progeny can still migrate to wound sites and become incorporated into tissues, but they fail to differentiate properly [9]. Therefore, the primary function of piwi in these animals may not be in stem-cell self-renewal but rather in potentiating the proper differentiation of neoblast progeny. The neoblast depletion RNAi phenotype observed might thus be due not to a specific failure of neoblast self-renewal but rather to exhaustion of the neoblast pool, resulting from an exceedingly high demand for differentiated cells as normal homeostasis fails.

\section{Future directions}

Characterizing the molecular properties of neoblasts and their non-neoblast progeny is unquestionably an important component of understanding the functioning of these remarkable stem cells. However, current research in stem-cell biology is providing increasing evidence that the behaviors and fates of stem cells are not inherent, cell-autonomous properties, but are instead critically dependent on external cues and feedback control [11]. Thus, to obtain a complete picture of how neoblasts function, it will be at least as important to understand the undoubtedly complex inputs that are integrated by these cells as they choose between alternative potential fates.

\section{Acknowledgements}

We thank members of Phil Newmark's lab for helpful discussion and Leo Shapiro for comments on the manuscript. AEB is supported by NSF grant IOS 0920502.

\section{Author details}

'Biology Department, University of Maryland, College Park, MD 20742, USA 2Department of Cell and Developmental Biology, University of Illinois at Urbana-Champaign, 601 South Goodwin Avenue, Urbana, IL 61801, USA

Published: 16 February 2010

\section{References}

1. Pellettieri J, Sánchez Alvarado A: Cell turnover and adult tissue homeostasis: From humans to planarians. Annu Rev Genet 2007, 41:83-105.

2. Reddien PW, Sánchez Alvarado A: Fundamentals of planarian regeneration. Annu Rev Cell Dev Biol 2004, 20:725-757.

3. Ladurner P, Rieger R, Baguñà J: Spatial distribution and differentiation potential of stem cells in hatchlings and adults in the marine platyhelminth Macrostomum sp.: a bromodeoxyuridine analysis. Dev Biol 2000, 226:231-241.

4. De Mulder K, Kuales G, Pfister D, Willems M, Egger B, Salvenmoser W, Thaler M, Gorny AK, Hrouda M, Borgonie G, Ladurner P: Characterization of the stem cell system of the acoel Isodiametra pulchra. BMC Dev Biol 2009, 9:69.

5. Paps J, Baguñà J, Riutort M: Lophotrochozoa internal phylogeny: new insights from an up-to-date analysis of nuclear ribosomal genes. Proc Biol Sci 2009, 276:1245-1254.

6. De Mulder K, Pfister D, Kuales G, Egger B, Salvenmoser W, Willems M, Steger J, Fauster K, Micura R, Borgonie G, Ladurner P: Stem cells are differentially 
regulated during development, regeneration, and homeostasis in flatworms. Dev Biol 2009, 334:198-212.

7. Eisenhoffer GT, Kang H, Sánchez Alvarado A: Molecular analysis of stem cells and their descendents during cell turnover and regeneration in the planarian Schmidtea mediterranea. Cell Stem Cell 2008, 3:327-339.

8. Pfister D, De Mulder K, Hartenstein V, Kuales G, G B, Marx F, Morris J, Ladurner P: Flatworm stem cells and the germ line: Developmental and evolutionary implications of macvasa expression in Macrostomum lignano. Dev Bio/ 2008, 319:146-159.

9. Reddien PW, Oviedo NJ, Jennings JR, Jenkin JC, Sánchez Alvarado A: SMEDWI- 2 is a PIWI-like protein that regulates planarian stem cells. Science 2005, 310:1327-1330.
10. Guo T, Peters AHFM, Newmark PA: A bruno-like gene is required for stem cell maintenance in planarians. Dev Cell 2006, 11:159-169.

11. Lander AD: The 'stem cell' concept: is it holding us back? J Biol 2009, 8:70

See other regeneration and stem cell articles $h t t p: / / j b i o l . c o m / c o n t e n t / 8 / 8 / 70$ and http://jbiol.com/content/9/2/15

doi:10.1186/jbiol223

Cite this article as: Bely AE, Sikes JM: Acoel and platyhelminth models for

stem-cell research. Journal of Biology 2010, 9:14. 